\section{Creutzfeldt-Jakob disease-like syndrome induced by gabapentin toxicity}

\author{
Vicky Chau,' Sadhana Prasad, ${ }^{2,3}$ \\ Dwight Stewart, ${ }^{2}$ George A. Heckman ${ }^{3,4}$ \\ 'University of British Columbia; \\ ${ }^{2}$ St.Mary's Hospital, Kitchener, Ontario; \\ ${ }^{3}$ McMaster University, Hamilton, Ontario; \\ ${ }^{3,4}$ University of Waterloo, Waterloo, \\ Ontario, Canada
}

\section{Abstract}

Patients with Creutzfeldt-Jakob disease (CJD) may exhibit characteristic abnormalities on the electroencephalogram (EEG). However, these abnormalities have been associated with a number of cases of drug toxicity. We report a case of CJD-like syndrome associated with gabapentin. A 78-year-old man was hospitalized for recurrent falls. Three months prior to admission, gabapentin was prescribed to treat symptoms of trigeminal neuralgia. The patient subsequently presented with a two-month history of worsening gait abnormalities, negative myoclonus, and cognitive impairment. The EEG showed diffuse background slowing with larger amplitude delta discharges, which at times appeared triphasic, raising the possibility of CJD. The gait abnormalities and myoclonus resolved and the EEG normalized after the gabapentin was discontinued. Several cases of drug-induced CJD-like syndrome have been reported, mainly presenting with cognitive impairment, myoclonus, Parkinsonism, and EEG abnormalities. This patient may have been predisposed to adverse neurological effects from gabapentin owing to age, concurrent renal insufficiency, and cardiac disease. We concluded that it is imperative to include drug toxicity in the differential diagnosis of patients presenting with clinical manifestations and EEG findings suggestive of CJD, particularly in the setting of advanced age and comorbidities.

\section{Introduction}

Creutzfeldt-Jakob disease (CJD) is a neurodegenerative disease caused by the accumulation of abnormal prion protein in the brain., ${ }^{1,2}$ While electroencephalography (EEG) of patients with CJD often demonstrates periodic biphasic or triphasic waves, numerous instances of CJD-like syndromes with similar EEG changes have been associated with drug toxicity. ${ }^{3-14}$ We report a case of a CJD-like syndrome associated with gabapentin.

\section{Case Report}

A 78-year-old man was hospitalized for falls. During the year prior to admission, he experienced a gradual decline in exercise tolerance and required a walking stick. His family noted no change in gait mechanics until two months prior to admission, when he began to experience repeated episodes of sudden, unpredictable loss of muscle tone affecting all limbs, and from which he recovered almost immediately. As a result, he would drop objects frequently and eventually was limited to 10 steps before his legs gave way and he fell. His family had not noticed any cognitive deficits, despite providing assistance with medication administration and transportation.

The patient and family reported a past medical history of stable ischemic cardiomyopathy, progressive chronic renal failure, diabetes mellitus, ischemic stroke with mild residual right-sided weakness, and localized prostate cancer treated with brachytherapy. He had long-standing trigeminal neuralgia treated with carbamazepine, and to which gabapentin (100 mg daily) was added five months prior to admission for worsening symptoms. His other medications included carbamazepine (200 mg four times daily), atenolol, amlodipine, lisinopril, furosemide, spironolactone, atorvastatin, aspirin, insulin, and nitroglycerin and oxazepam as needed.

The neurological examination was remarkable for negative myoclonus of his upper and lower extremities during sustained posture. He was unable to hold an object in his hands or stand for any length of time, precluding formal gait assessment. Power in all limbs was normal, with minimally increased tone in the right arm. Deep tendon reflexes were normal and plantar reflexes were flexor. Cranial nerves were unremarkable. Occasional fasciculations were noted in the distal extremities. Cerebellar testing was not performed. The rest of his medical examination was unremarkable. Subsequent cognitive testing revealed a score of 74/100 on the Modified Mini Mental State (3MS) and inability to correctly place the hands on the Clock Drawing Test (CDT), with deficits of executive function, visual spatial, and verbal fluency.

The complete blood count and electrolytes were normal. Random blood glucose was 14.4 $\mathrm{mmol} / \mathrm{L}$, serum creatinine was $214 \mu \mathrm{mol} / \mathrm{L}$, and urea was $35.6 \mathrm{mmol} / \mathrm{L}$. The electrocardiogram showed first-degree heart block. A chest X-ray revealed no acute abnormalities. Computed tomography scans of the brain demonstrated old lacunar infarcts in the left basal ganglia and right cerebellum. The EEG showed diffuse background slowing with larger amplitude delta discharges, which at times appeared triphasic and were interpreted as consistent
Correspondence: George A. Heckman, Schlegel Research Chair in Geriatric Medicine, Associate Professor, Department of Health Studies and Gerontology, Faculty of Applied Health Sciences, University of Waterloo, 200 University Avenue West, Waterloo, ON, N2L 3G1, Canada.

E-mail: ggheckma@uwaterloo.ca

Key words: gabapentin, adverse drug event, Creutzfeldt-Jakob disease, elderly.

Acknowledgements: the authors would like to acknowledge Jim Engle for his technical support in interpreting and analyzing the electroencephalogram.

Contributions: VC, SP, DS, GH analyzed, interpreted patient data, and were all major contributors in writing the manuscript. All authors read and approved the final manuscript.

Conflict of interest: the authors report no conflicts of interest.

Received for publication: 18 November 2009.

Revision received: 18 May 2010.

Accepted for publication: 23 May 2010.

This work is licensed under a Creative Commons Attribution 3.0 License (by-nc 3.0)

(C) Copyright V. Chau et al., 2010

Licensee PAGEPress, Italy

Ageing Research 2010; 2:e3

doi:10.4081/ar.2010.e3

with periodic sharp wave complexes (PSWC) of CJD (Figure 1). Electromyography indicated mild to moderate generalized sensorimotor neuropathy, consistent with a diabetic neuropathy and likely responsible for the fasciculations observed.

Gabapentin was discontinued, as its initiation had preceded neurological deterioration. Within 48 hours, the myoclonus had improved such that he could hold objects and walk with a cane. An EEG two months later was normal.

\section{Discussion}

CJD is a rapidly progressive neurodegenerative disorder often characterized by myoclonus and characteristic PSWC on EEG (Table 1)., ${ }^{1,2}$ Definitive diagnosis requires post-mortem examination. PSWC occur in about two-thirds of patients with CJD, and may not be present early in the disease course. ${ }^{15}$ The sensitivity and specificity of PSWC of CJD are approximately $64 \%$ and $91 \%$, respectively, with other etiologies including hepatic, anoxic, and toxic encephalopathies. ${ }^{16}$

A search of the English literature identified approximately 15 cases of a CJD-like syndrome 


\section{Table 1. MRI-CJD consortium criteria for sporadic Creutzfeldt-Jakob disease.}

\section{Clinical}

1) Rapidly evolving dementia

2) Cerebellar and/or visual signs and symptoms

3) Extrapyramidal and/or pyramidal signs and symptoms

4) Akinetic mutism

II. Tests

1. Typical periodic sharp wave complexes (PSWC) with triphasic morphology on EEG

2. Detection of 14-3-3 protein in cerebrospinal fluid examination (in patients with a disease duration of less than $2 \mathrm{yr}$ )

3. High signal abnormalities in the caudate nucleus and putamen or at least two cortical regions (temporal-parietal-occipital) either in DWI or FLAIR

\section{Diagnostic criteria for sporadic Creutzfeldt-Jakob disease}

Definite Neuropathological examination or detection of scrapie prion protein $\left(\mathrm{PrP}^{\mathrm{Ps}}\right)$ by Western blot analysis of brain bioptic or autoptic specimens

Probable Two out of I and at least one out of II (above)

Possible Two out of I and duration of less than $2 \mathrm{yr}$

Note: myoclonus, previously considered a criterion for probable CJD in the WHO criteria, has been dropped by the MRI-CJD consortium.

Table 2. Summary of drug-induced Creutzfeldt-Jakob-like syndromes.

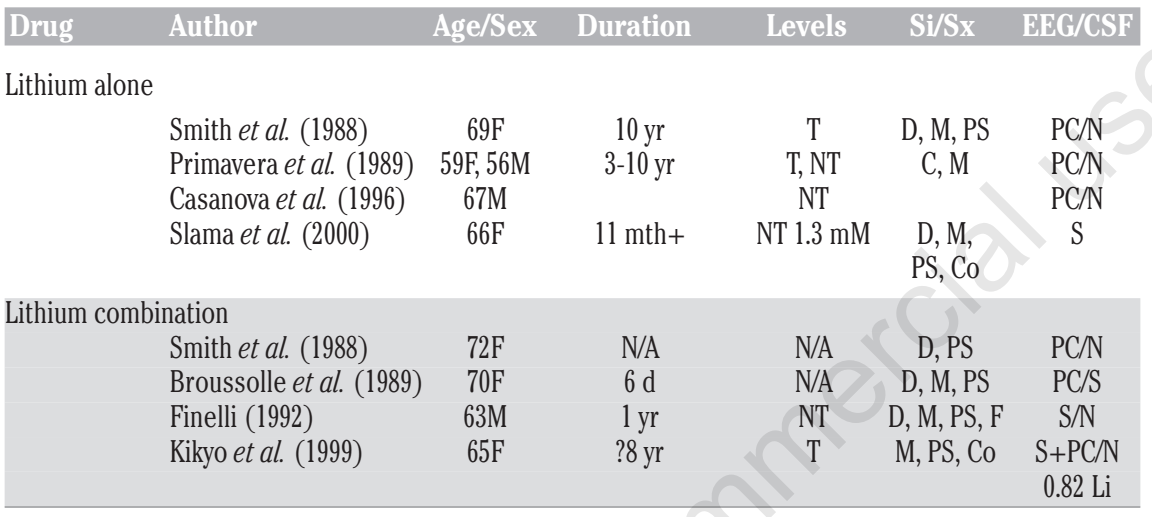

\begin{tabular}{lcccccc}
\hline Mianserin & & & & & \\
& Koponen et al. (1990) & $61 \mathrm{M}$ & N/A & N/A & C & PC, S/N \\
Amitriptyline & & $68 \mathrm{~F}$ & $2 \mathrm{wk}$ & Man359 & M, C & PC, S/N \\
& & & & & & \\
& Foerstl et al. (1989) & $62 \mathrm{~F}$ & N/A & NT & D, M, ?PS & PC/N \\
\hline
\end{tabular}

Carbamazepine

$\begin{array}{lllll}\text { Horvath et al. }(2005) \quad 71 \mathrm{M} & 4 \mathrm{mth} & \mathrm{T} & \mathrm{D}, \mathrm{M}, \mathrm{PS} \quad \mathrm{PC} / \mathrm{N}\end{array}$

\begin{tabular}{|c|c|c|c|c|c|c|}
\hline Polypharmacy & Waldman (1990) & $68 \mathrm{~F}$ & N/A & N/A & D. M.PS & Minimal \\
\hline
\end{tabular}

Ceftazidime

\begin{tabular}{lllllll} 
Chuang et al. (2004) & $76 \mathrm{~F}$ & $8 \mathrm{~d}$ & $\mathrm{~T}$ & $\mathrm{C}, \mathrm{M}$ & S, PSIDD \\
\hline
\end{tabular}

T, toxic; NT, nontoxic; D, dementia; M, myoclonus; PS, Parkinsonian syndrome; C, confusion; Co, coma; F, fasciculation; PC, periodic complexes; $\mathrm{N}$, normal; S, slowing; PSIDD, periodic short-interval diffuse discharge.

associated with mianserin, ${ }^{3}$ amitryptyline, ${ }^{4}$ nortriptyline,${ }^{5}$ carbamazepine, ${ }^{6}$ polypharmacy, ${ }^{7}$ ceftazidime, ${ }^{8}$ and lithium, either alone ${ }^{9-12}$ or in combination with levodopa, ${ }^{7,13}$ levomepromazine, or phenobarbitone ${ }^{14}$ (Table 2). Clinical features most commonly included cognitive impairment, myoclonus, and Parkinsonism, and less frequently fasciculations and coma. Most patients demonstrated EEG abnormali- ties consistent with CJD. There have been no reports of CJD-like syndromes associated with gabapentin toxicity.

Gabapentin has been used in the treatment of seizures, epilepsy, neuropathic pain, and mental illness, as it is considered safe with minimal toxicity. It is a structural analog of gaba-aminobutyric acid (GABA) able to cross the blood brain barrier and enhance

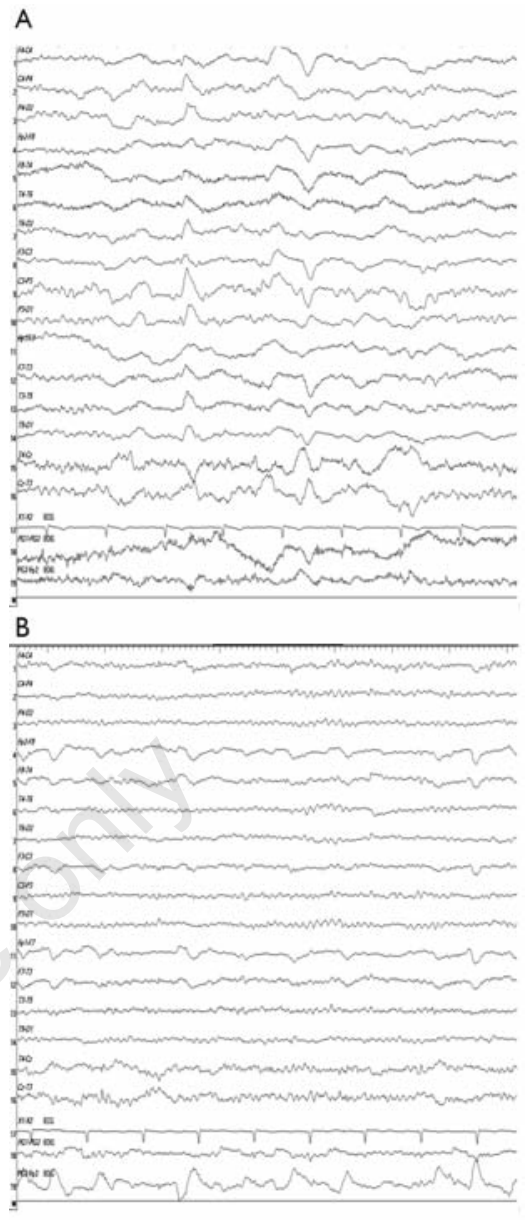

Figure 1. (A) EEG showed diffuse slowing of background rhythms with periodic sharp waves of $1.5-2.5 \mathrm{~Hz}$ in the anterior and frontal areas. These at times appeared to assume a triphasic pattern suggesting the possibility of spongiform encephalopathy. (B) EEG two months after discontinuation of gabapentin showed a normal recording with disappearance of periodic complexes.

GABAergic transmission. ${ }^{17}$ Elimination is dependent solely on renal excretion where its clearance is linearly proportional to creatinine clearance. Adverse effects of gabapentin reported include ataxia, choreoathetosis, dyskinesia, dystonia, asterixis, myoclonus, myopathy, and neurological toxicities. The risk for adverse effects is increased regardless of dosage in the setting of impaired renal function, poor cardiac function, or in the elderly. ${ }^{14}$

We have described a patient with a CJD-like syndrome characterized by gait abnormalities, negative myoclonus, cognitive impairment, and EEG tracings suggestive of CJD. Although his family did not report significant deficits, cognitive dysfunction may not have been apparent as most of the patient's independent activities of daily living were managed by the family. The absence of reported cognitive symptoms does 
not rule out CJD. In one series of 230 neuropathologically confirmed cases of CJD, $36 \%$ had not presented with cognitive symptoms. ${ }^{18}$ However, simply asking family members about cognitive changes is not a reliable way to detect dementia. ${ }^{19}$ Furthermore, cognitive impairment was present on objective testing. On the 3MS broadened scoring system, the standard cut-off point of $79 / 100$ or less indicates cognitive impairment. ${ }^{20}$ An abnormal clock is also specific for significant cognitive impairment. ${ }^{21}$

The resolution of the syndrome and EEG normalization on discontinuation of gabapentin suggests that this syndrome may have been a manifestation of gabapentin toxicity. This patient may have been predisposed to adverse neurological effects from gabapentin owing to age, concurrent renal insufficiency, and cardiac disease. Although carbamazepine can induce similar reactions, this patient was ingesting a lower dose than the one associated with a CJDlike syndrome. ${ }^{6}$ While a pharmacokinetic interaction between carbamazepine and gabapentin is unlikely because of differing metabolic pathways and the lack of significant protein binding of gabapentin, a pharmacodynamic interaction, in which the gabapentin potentiated carbamazepine toxicity, cannot be ruled out. ${ }^{22}$

\section{Conclusions}

We conclude that it is imperative to consider medication toxicity in patients presenting with new neurological symptoms, including clinical manifestations and EEG findings suggestive of CJD, particularly in the setting of advanced age and comorbidities that may increase the risk of adverse drug reactions.

\section{References}

1. Zerr I, Kallenberg K, Summers DM, et al. Updated clinical diagnostic criteria for sporadic Creutzfeldt-Jakob Disease. Brain 2009;132:2659-68.

2. World Health Organization. Consensus on criteria for diagnosis of sporadic CJD. Wkly Epidemiol Rec 1998;73:361-5.

3. Koponen H, Honkonen S, Partanen J, et al. Epileptic attack, delirium, and a Creutzfeldt-Jakob-like syndrome during mianserin treatment. Neuropsychobiology 1990;23:164-8.

4. Foerstl J, Hohagen F, Hewer W, et al. Another case of Creutzfeldt-Jakob-like syndrome due to antidepressant toxicity. J Neurol Neurosurg Psychiatry 1989;52:920.

5. Finelli PF. Drug-induced CreutzfeldtJakob-like syndrome. J Psychiatr Neurosci 1992;17:103-5.

6. Horvath J, Coeytaux A, Jallon P, et al. Carbamazepine encephalopathy masquerading as Creutzfeldt-Jakob disease. Neurology 2005;65:650-1.

7. Waldman AJ. A Creutzfeldt-Jakob-like syndrome due to polypharmacy. South Med J 1990;12:1493.

8. Chuang CL, Chen KP, Kwan SY, et al. Creutzfeldt-Jakob-like EEG in a patient with end-stage renal failure. Nephrol Dial Transplant 2004;19:252-4.

9. Smith SJ, Kocen RS. A Creutzfeldt-Jakoblike syndrome due to lithium toxicity. J Neurol Neurosurg Psychiatry 1988;51:1203.

10. Primavera A, Brusa G, Poeta MG. A Creutzfeldt-Jakob-like syndrome due to lithium toxicity. J Neurol Neurosurg Psychiatry 1989;52:423.

11. Casanova B, de Entrambasaguas M, Perla C, et al. Lithium-induced Creutzfeldt-
Jakob syndrome. Clin Neuropharmacol 1996;19:356-9.

12. Slama M, Masmoudi K, Blanchard N, et al. A possible case of lithium intoxication mimicking Creutzfeldt-Jakob syndrome. Pharmacopsychiatry 2000;33:145-6.

13. Broussolle E, Setiey A, Moene Y, et al. Reversible Creutzfeldt-Jakob-like syndrome induced by lithium plus levodopa treatment. J Neurol Neurosurg Psychiatry 1989:52:686-7.

14. Kikyo H, Furukawa T. Creutzfeldt-Jakoblike syndrome induced by lithium, levomepromazine, phenobarbitone. J Neurol Neurosurg Psychiatry 1999;66:802-3.

15. Steinhoff BJ, Zerr I, Glatting M, et al. Diagnostic value of periodic complexes in Creutzfeldt-Jakob disease. Ann Neurol 2004;56:702-8.

16. Wieser HG, Schindler K, Zumsteg D. EEG in Creutzfeldt-Jakob disease. Clin Neurophysiol 2006;117:935-51.

17. Sill GJ. The mechanisms of action of gabapentin and pregabalin. Curr Opin Pharmacol 2006;6:108-13.

18. Brown P, Cathala F, Castaigne P, et al. Creutzfeldt-Jakob disease: Clinical analysis of a consecutive series of 230 neuropathologically verified cases. Ann Neurol 1986;20:597-602.

19. Watson LC, Lewis CL, Fillenbaum GG. Asking family about memory loss. Is it helpful? J Gen Intern Med 2005;20:28-32.

20. Teng EL, Chui HC. The Modified MiniMental State (3MS) Examination. J Clin Psychiatry 1987;48:314-8.

21. Pinto E, Peters R. Literature review of the Clock Drawing test as a tool for cognitive screening. Dement Geriatr Cogn Disord 2009;27:201-13.

22. LaRoche SM. A new look at the second generation antiepileptic drugs: a decade of experience. Neurologist 2007;13:133-9. 